\title{
REMOCIÓN DE LIGNINA EN EL PRETRATAMIENTO DE CASCARILLA DE ARROZ POR EXPLOSIÓN CON VAPOR
}

\author{
Ricardo Aaron Naveda Rengifo*b, Paola Aurelia Jorge Montalvo, \\ Lisveth Flores del Pino ${ }^{a}$, Lizardo Visitación Figueroa ${ }^{\mathrm{a}, \mathrm{b}}$
}

\begin{abstract}
RESUMEN
El presente estudio evaluó la remoción de la lignina de la cascarilla de arroz, mediante la explosión con vapor a diferentes condiciones de severidad. El proceso de remoción de lignina evaluado como lignina Klason fue realizado en muestras con pretratamiento a presiones de 0,4; 0,6; 0,8 y 1,0 MPa, tiempos de 6, 8, 10 y 12 minutos con una descompresión de entre 5 a 7 segundos; se logró una remoción de lignina desde 18,02 \% hasta $12,7 \%$ a 1,0 MPa y 12 min de compresión, factor de severidad de 3,3. La evaluación por FTIR de la cascarilla sin pretratamiento, con pretratamiento a $0,4 \mathrm{MPa}$ y $12 \mathrm{~min}, 1,0 \mathrm{MPa}$ y $12 \mathrm{~min}$, nos presenta una reducción de la transmitancia generada por los picos que corresponden a la presencia de lignina $-\mathrm{OH}\left(2920 \mathrm{~cm}^{-1}\right), \mathrm{C}-\mathrm{H}$ y C-O (1320 y $\left.1360 \mathrm{~cm}^{-1}\right), \mathrm{C}-\mathrm{O}-\mathrm{C}$ a $\left(1060 \mathrm{~cm}^{-1}\right)$ entre otros. La evaluación por microscopia de barrido electrónico (SEM) demostró el grado de remoción del tejido superficial de la cascarilla de arroz asociado con la remoción de lignina. El pretratamiento por explosión con vapor de la cascarilla de arroz mejora el potencial de hidrólisis de la cascarilla de arroz para la formación de azúcares reductores que puedan ser aprovechados en la producción de etanol G-2, debido a la remoción de lignina y exposición de la celulosa y hemicelulosa.
\end{abstract}

Palabra clave: explosión con vapor, cascarilla de arroz, lignina Klason, FTIR, SEM

\section{LIGNINE REMOTION FROM RICE HUSK PRETREATMENT BY STEAM EXPLOSION}

\begin{abstract}
The present study evaluated the removal of lignin from the rice husk, by means of steam explosion at different severity conditions. The process of lignin removal was evaluated as lignin Klason was performed in samples with pretreatment at pressures of $0.4 ; 0.6 ; 0.8$ and $1.0 \mathrm{MPa}$, times of $6,8,10$ and 12 minutes with a decompression of between 5 to 7 seconds;

${ }^{a}$ Centro de Investigación en Química, Toxicología y Biotecnología Ambiental CIQTOBIA Facultad de Ciencias, Universidad Nacional Agraria La Molina, Lima - Perú

${ }^{\text {b }}$ Centro Modelo de Tratamiento de Residuos CEMTRAR - Universidad Nacional Agraria La Molina, Lima - Perú *aanaveda@gmail.com
\end{abstract}


it is a lignin removal from $18.02 \%$ up to $12.7 \%$ at $1.0 \mathrm{MPa}$ and $12 \mathrm{~min}$ compression, factor of 3.3 severity. The evaluation by FTIR of the husk without pretreatment, with pretreatment at $0.4 \mathrm{MPa}$ and $12 \mathrm{~min}, 1.0 \mathrm{MPa}$ and $12 \mathrm{~min}$, shows a reduction in the transmission generated by the peaks corresponding to the presence of lignin $-\mathrm{OH}\left(2920 \mathrm{~cm}^{-1}\right), \mathrm{CH}$ and $\mathrm{CO}(1320$ and $\left.1360 \mathrm{~cm}^{-1}\right)$, COC a $\left(1060 \mathrm{~cm}^{-1}\right)$ among others. The promover per screening electronic microscopy (SEM) showed the degree of introducción Sud cantidades of the surface of the rice associated with theollowing of the lignin explicar. The pretreatment of the steam explosion of the rice husk improves the potential of rice husk hydrolysis for the formation of reducing sugars that can be exploited in the production of G-2 ethanol, due to the removal of lignin and the exposure of cellulose and hemicellulose.

Key words: steam explosion, rice husk, lignin klason, FTIR, SEM.

\section{INTRODUCCIÓN}

La cascarilla de arroz es un residuo lignocelulósico que se obtiene de la producción de arroz a partir de la planta Oryza sativa. En el Perú, según el Instituto de Innovación Agraria (INIA), es el primer producto en área sembrada y cosechada 0,38 millones de hectáreas durante el 2014ํ․ De acuerdo a la FAO, en el 2014 se sembró 162,7 millones de hectáreas produciendo aproximadamente 741 millones de toneladas de arroz que contiene entre $20-21 \%$ de cascarilla ${ }^{2}$, generando aproximadamente 150 millones de toneladas de este residuo que tiene un buen potencial para el desarrollo en la producción de etanol $2 \mathrm{G}$.

Los residuos lignocelulósicos de cascarilla de arroz compuestos de celulosa, hemicelulosa y lignina, pueden ser reaprovechados para la hidrólisis de celulosa como fuente principal de azúcares. Se requiere remover las barreras naturales que impiden el ataque microbiano, así como la minimización de la degradación de los azúcares a compuestos inhibitorios ${ }^{3,4,5}$.

La lignina se encuentra unida a la celulosa y hemicelulosa, su diversidad estructural compuesta principalmente por tres tipos de fenilpropano (p-hidroxifenil, guaiacil, y siringil) unidos entre ellos por enlaces éter y carbono-carbono; así como su heterogenicidad química dificultan su biodigestibilidad $^{6,7}$ e impiden el ataque microbiano de la celulosa y hemicelulosa.

El pretratamiento por explosión con vapor es un método que involucra parámetros críticos del proceso como el uso de altas temperaturas (o presiones) en un corto tiempo de tratamiento seguido de descomprensión rápida que modifica la estructura de la pared celular de la cascarilla de arroz, dejando un material de color marrón, indicativo de la hidrólisis de hemicelulosa fácilmente soluble en agua; una fracción de un material insoluble en agua compuesto por celulosa, residuos de hemicelulosa y lignina ${ }^{8,9}$.

En el pretratamiento por explosión con vapor de los residuos lignocelulosicos el pH del material residual y del lixiviado puede caer a valores ácidos, esto es atribuido a la formación de ácidos orgánicos, como el ácido acético formado a partir de los grupos acetilo de la 
hemicelulosa ${ }^{10}$. Esta acidez produce la autohidrólisis de la hemicelulosa ${ }^{9}$. Dependiendo de la severidad del tratamiento (Ro) se pueden producir dos tipos de reacciones: la depolimerización por hidrólisis ácida y la condensación - repolimerización de la lignina ${ }^{8,9,10,11}$.

$$
R o=\log \left(t x e^{\frac{T-100}{14,75}}\right)
$$

Donde Ro es el factor de severidad, $\mathrm{t}$ es el tiempo en minutos y $\mathrm{T}$ es la temperatura en ${ }^{\circ} \mathrm{C}$. La transformación de la estructura química de la lignina puede ser evaluada por la aparición, disminución o desaparición de grupos funcionales y enlaces característicos como los grupo hidroxilo $-\mathrm{OH}^{12,13}$, carbonilo $\mathrm{C}=\mathrm{O}$ que puede corresponder a los ésteres alifáticos encontrados en la lignina y la hemicelulosa ${ }^{14}$, así como los enlaces $\mathrm{C}-\mathrm{O}$ unidos a poliscaridos o anillos aromáticos en residuos de lignina, entre otros ${ }^{15}$.

El objetivo del presente trabajo fue evaluar la influencia del pretratamiento con explosión de vapor a temperatura o presiones bajas sobre el contenido de lignina en la estructura de la cascarilla de arroz, como paso previo para la hidrólisis enzimática.

\section{PARTE EXPERIMENTAL}

\section{Toma de muestra y acondicionamiento}

Los residuos de cascarilla de arroz semilla de la planta Oryza sativa, fueron recolectados de la ciudad de Chiclayo producidos por la Asociación Peruana de Molineros de Arroz (APEMA) y Molino Sudamérica, en mayo del 2017. Estos fueron secados a temperatura ambiente manteniendo una humedad entre 8 a $12 \%$. Las dimensiones de las muestras fueron 9,2 - 10,7 $\mathrm{mm}$ de largo, 2,1 - 3,8 $\mathrm{mm}$ de ancho por 0,8 a 1,7 $\mathrm{mm}$ de espesor, resultados similares a los obtenidos por otros estudios ${ }^{16,17}$, debido a esto los residuos no fueron triturados por tener un tamaño cercano a $7 \mathrm{~mm}$ adecuado para el pretratamiento por explosión con vapor.

\section{Pretratamiento de explosión con vapor:}

La explosión de vapor fue realizada utilizando un equipo que está compuesto de un tanque de acero inoxidable de $15 \mathrm{~L}$ acoplado a un generador eléctrico de vapor diseñado para el presente proyecto. Se colocó $300 \mathrm{~g}$ de residuos de cascarilla de arroz con una humedad promedio de $8,78 \%$, el tanque fue rellenado con vapor saturado a presiones de 0,$4 ; 0,6 ; 0,8$ y $1,0 \mathrm{MPa}$ manteniendo una temperatura entre $130-180{ }^{\circ} \mathrm{C}$, los tiempos de reacción utilizados fueron 6, 8, 10 y $12 \mathrm{~min}$, el tiempo de descompresión estuvo entre 5 a 7 s. Luego de los tratamientos se midio el valor del pH del líquido generado por la explosión de vapor. El diseño utilizado en los pretratamientos fue bifactorial completo, donde la unidad experimental fue la cascarilla de arroz, los factores fueron la presión ( $\mathrm{MPa}$ ) y el tiempo de residencia (minutos), y la variable respuesta fue el contenido de lignina de la cascarilla de arroz (porcentaje). 


\section{Caracterización química}

\section{Determinación de la lignina insoluble en ácido (LIA)}

El contenido de lignina en las muestras fue determinado conforme a lo establecido por el reporte técnico de la National Renewable Energy Laboratory OF United States NREL/TP510-42618. El principio de este método se basa en someter a la biomasa al ataque de un ácido fuerte que hidrolice y disuelva todos los carbohidratos, quedando solo la lignina en la fase sólida. Bajo este principio, la lignina puede ser cuantificada y clasificada de dos maneras, lignina insoluble en ácido (LIA) y lignina soluble en ácido (LSA). La primera es aquella parte sólida que queda luego del ataque con el ácido y se cuantifica por gravimetría, mientras que la segunda es aquella que llegó a ser solubilizada y pasó a formar parte del lixiviado y puede ser cuantificada mediante cromatografía. Para efectos de la presente investigación, se considerará solo la LIA conocida como lignina Klason.

\section{Microscopia de barrido electrónico (SEM)}

Las muestras fueron observadas por la parte interna y externa de su superficie utilizando microscopia electrónica de barrido (SEM), dos muestras tratadas por explosión con vapor CT-1 (4 Bar, $12 \mathrm{~min}$ ) y CT-2 (10 Bar, $12 \mathrm{~min}$ ) así como una muestra no tratada ST. Las imágenes fueron obtenidas utilizando un microscopio electrónico modelo Q250 Analytical. Para el análisis las muestras fueron secadas y cubiertas con una película de oro, se utilizó una aceleración de voltaje de $20 \mathrm{kV}$. Las imágenes fueron obtenidas a 75 y 370 magnificaciones.

\section{Espectroscopia infrarroja con transformada de Furier}

Para examinar los cambios en la estructura química de las muestras sin y con pretratamiento ST, CT-1 y CT-2 fueron analizadas utilizando un Espectrofotómetro Infrarrojo con Transformada de Furier (FTIR) modelo Nicolet iS10, en el espectro entre 4000 a $600 \mathrm{~cm}^{-1}$.

\section{RESULTADOS Y DISCUSIÓN}

\section{Caracterización química}

El contenido de lignina en las muestras de cascarilla de arroz encontrada en el presente estudio fue de $18,02 \%$, este valor se encuentra en el rango $(18,2-24,6 \%)^{18}$, es superior a $10,9 \%{ }^{19}$ e inferior a $20,4-33,7 \%^{20}$, las diferentes especies utilizadas en los cultivos de arroz son responsables de estas variaciones. La humedad y el contenido de cenizas determinada fueron 8,78 y $18,75 \%$, respectivamente se encuentran en el rango de 4,55$10,57 \%$ y $9,29-30,18 \%$, determinado por otros estudios 2 . 
Tabla 1. Composición química de la cascarilla de arroz.

\begin{tabular}{cc}
\hline Componente & Contenido \% \\
\hline Lignina & $18,02 \pm 0,87$ \\
Humedad & $8,78 \pm 0,41$ \\
Cenizas & $18,45 \pm 0,38$ \\
Extractivos & $1,98 \pm 0,01$
\end{tabular}

Nota: Resultados \pm Desviación Estándar para dos muestras por triplicado

El contenido de extractivos en la muestra de cascarilla de arroz fue 1,98 \%, está formado de sustancias de bajo peso molecular, carbohidratos solubles, flavonoides, terpenos, lignanos entre otras sustancias ${ }^{8}$.

\section{Pretratamiento por explosión con vapor}

\section{Efecto sobre el pH}

El pH del lixiviado de cascarilla de arroz después de la explosión con vapor disminuye hasta valores entre 4,2 - 4,4 con el incremento del factor de severidad. Esto se debe a la rápida formación de ácidos orgánicos de cadena corta como el ácido acético generado a partir de los grupos acetilo de la hemicelulosa ${ }^{21,10}$. El incremento del tiempo de exposición al vapor disminuye ligeramente el valor del $\mathrm{pH}$ independientemente del valor de la temperatura, debido a la mayor oxidación de la materia orgánica termalmente descompuesta ${ }^{8}$. Los valores bajos de $\mathrm{pH}$ promueven la autohidrólisis de la hemicelulosa y deshidratación de los azúcares liberados que pueden producir hidroximetilfurfural ${ }^{8,9}$. 
Tabla 2. Valor de $\mathrm{pH}$, material extractable y contenido de lignina en el material sólido remanente después del pretratamiento de la cascarilla de arroz por explosión con vapor, bajo diferentes condiciones de temperatura y tiempos de residencia.

\begin{tabular}{|c|c|c|c|c|c|c|}
\hline $\begin{array}{c}\text { Presión } \\
\mathrm{MPa}\end{array}$ & $\begin{array}{c}\text { Temperatura } \\
{ }^{\circ} \mathrm{C}\end{array}$ & $\begin{array}{c}\text { Tiempo } \\
\text { min }\end{array}$ & $\begin{array}{l}\text { Factor de } \\
\text { Severidad }\end{array}$ & $\mathrm{pH}$ & $\begin{array}{c}\% \\
\text { Extractables }\end{array}$ & \% Lignina \\
\hline 0,4 & 129,1 & 6 & 1,6 & $6,8 \pm 0,8$ & $2,0 \pm 0,7$ & $15,9 \pm 0,1$ \\
\hline 0,4 & 129,1 & 8 & 1,8 & $6,9 \pm 0,9$ & $2,0 \pm 0,7$ & $15,6 \pm 1,1$ \\
\hline 0,4 & 129,1 & 10 & 1,9 & $6,8 \pm 0,7$ & $2,6 \pm 0,2$ & $16,9 \pm 0,7$ \\
\hline 0,4 & 129,1 & 12 & 1,9 & $6,2 \pm 0,3$ & $3,4 \pm 0,8$ & $17,4 \pm 0,2$ \\
\hline 0,6 & 147,4 & 6 & 2,2 & $7,0 \pm 0,4$ & $2,4 \pm 0,0$ & $14,9 \pm 1,5$ \\
\hline 0,6 & 147,4 & 8 & 2,3 & $6,2 \pm 0,0$ & $2,9 \pm 0,2$ & $14,3 \pm 0,3$ \\
\hline 0,6 & 147,4 & 10 & 2,4 & $6,7 \pm 0,6$ & $2,2 \pm 0,0$ & $17,1 \pm 0,5$ \\
\hline 0,6 & 147,4 & 12 & 2,5 & $5,9 \pm 0,1$ & $3,6 \pm 0,0$ & $15,0 \pm 1,0$ \\
\hline 0,8 & 161,9 & 6 & 2,6 & $6,4 \pm 0,5$ & $3,5 \pm 0,3$ & $14,6 \pm 0,4$ \\
\hline 0,8 & 161,9 & 8 & 2,7 & $5,4 \pm 0,9$ & $3,0 \pm 0,0$ & $13,7 \pm 0,6$ \\
\hline 0,8 & 161,9 & 10 & 2,8 & $5,0 \pm 0,4$ & $3,0 \pm 1,1$ & $14,9 \pm 0,6$ \\
\hline 0,8 & 161,9 & 12 & 2,9 & $3,8 \pm 0,9$ & $3,5 \pm 0,7$ & $13,5 \pm 0,7$ \\
\hline 1,0 & 174,1 & 6 & 3,0 & --- & $6,2 \pm 0,3$ & $14,2 \pm 0,4$ \\
\hline 1,0 & 174,1 & 8 & 3,1 & $4,4 \pm 0,1$ & $6,2 \pm 0,5$ & $13,4 \pm 0,8$ \\
\hline 1,0 & 174,1 & 10 & 3,2 & $4,4 \pm 0,2$ & $4,5 \pm 0,0$ & $12,8 \pm 0,6$ \\
\hline 1,0 & 174,1 & 12 & 3,3 & $4,2 \pm 0,0$ & $6,1 \pm 0,5$ & $12,7 \pm 1,2$ \\
\hline
\end{tabular}

Nota: Resultados \pm Desviación Estándar para dos muestras por triplicado 


\section{Efecto sobre el contenido de extraíbles y lignina}

En el rango de presión utilizada en la presente investigación se observa que al incrementar el tiempo a presiones entre 0,4 y $0,6 \mathrm{MPa}$ el porcentaje de lignina inicialmente (8 primeros minutos) se reduce por la depolimerización de la lignina generada por la autohidrólisis (escisión del enlace $\beta$-O-4 éter en medio ácido), seguida por un incremento de este (minuto 12) debido a reacciones de condensación y repolimerización entre los productos de descomposición de la hemicelulosa y la lignina ${ }^{11,10}$, también se observa un incremento de los extraíbles debido al incremento de los productos de descomposición de la hemicelulosa y lignina. En el rango de presiones entre 0,8 y 1,0 MPa el porcentaje de lignina disminuye con el tiempo debido a la depolimerización de la lignina, se incrementa el contenido de extraíbles por la formación de productos de descomposición de la hemicelulosa y depolimerización de la lignina. En el rango de temperaturas y presiones trabajados se observa una reducción del porcentaje de lignina con el incremento del facto de severidad debido a que se favorecen la reacción de depolimeración de la lignina en lugar de la de condensación.

\section{Espectroscopía infrarroja por transformada de Fourier (FTIR)}

Los espectros FTIR de muestras tratadas CT-1 y CT-2 de cascarilla de arroz bajo diferentes tratamientos, presentan disminución de la absorción dominante con respecto a la muestra no tratada ST en los picos: a $3340 \mathrm{~cm}^{-1}$ atribuidas a vibraciones tipo strech de los grupos $-\mathrm{OH}$ asociados a la estructura de la celulosa y lignina; a $2920 \mathrm{~cm}^{-1}$ atribuida a C-H asociado a la estructura de la celulosa y hemicelulosa ${ }^{12,13}$; a $1739 \mathrm{~cm}^{-1}$ atribuidos a la vibración strech del grupo carbonilo $(\mathrm{C}=\mathrm{O})$, asociado a los ésteres alifáticos de lignina y la hemicelulosa ${ }^{14}$; a 1320 y $1360 \mathrm{~cm}^{-1}$ atribuidos a los grupos C-H y C-O de anillos aromáticos de polisacáridos o residuos de lignina; a $1060 \mathrm{~cm}^{-1}$ atribuido a $\mathrm{C}-\mathrm{O}-\mathrm{C}$ del anillo piranósico vibración tipo esqueleto, relacionado a la lignina, celulosa y hemicelulosa ${ }^{15}$. El tratamiento CT-2 presentó la mayor reducción en la transmitancia de los picos esto se asocia a un menor contenido de lignina.

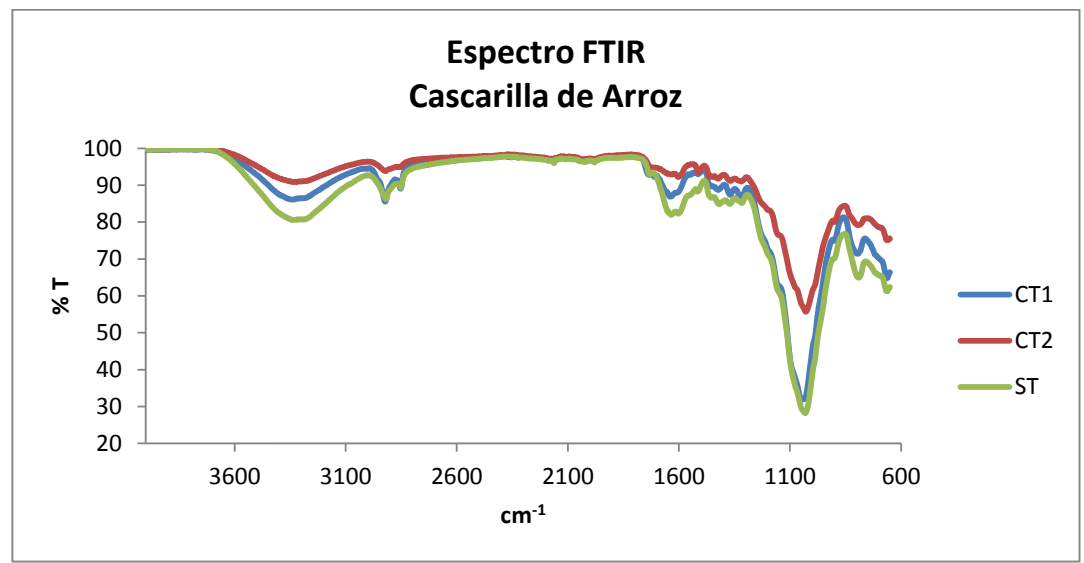

Figura 1. Espectro FTIR de la cascarilla de arroz, sin y con tratamiento por explosión de vapor. 


\section{Microscopia de barrido electrónico}

La morfología típica de la cascarilla de arroz determinada por la microscopia de barrido electrónica (SEM) mostró que la superficie externa tiene forma globular con células epidérmicas dispuestas en surcos lineales, con crestas salpicadas con protuberancias globulares y con menor frecuencia con pelos (tricomas) similar a lo reportado por otros estudios $^{22}$. Se observa que la muestra sin tratamiento ST presenta una superficie lisa y regular tanto en las protuberancias globulares como en los pelos. La muestra con tratamiento suave CT-1 de forma similar a la muestra ST presenta una superficie lisa con algunas fracturas en el pelo. Por otro lado la muestra con tratamiento con mayor factor de severidad presenta mayor remoción de la superficie lisa (desaparición de la lignina) tanto en la protuberancia globular como el pelo, también se observa mayor contenido de restos de la superficie como partículas sobre la superficie, ver figura 2. Esto es un claro indicativo de la remoción de la lignina de la pared celular de la cascarilla de arroz, dejando expuesta las cadenas de celulosa y hemicelulosa mejorando su potencial para posterior hidrólisis ácida o enzimática de la cascarilla de arroz.
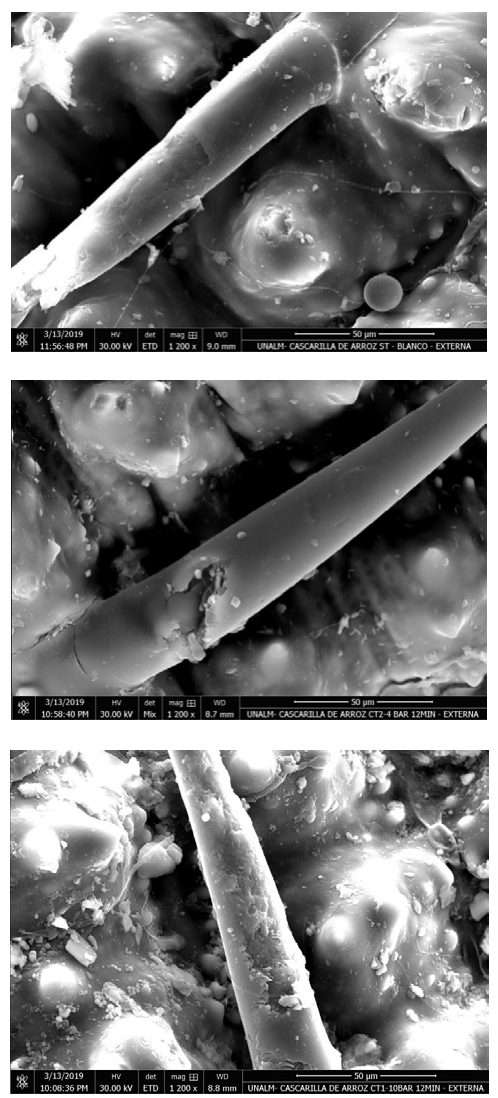

Figura 2. Cascarilla de arroz cara externa sin tratamiento (superior), con tratamiento por explosión con vapor $0,4 \mathrm{MPa} 12$ minutos (medio), con vapor 1,0 MPa 12 minutos (inferior). 


\section{CONCLUSIONES}

El pretratamiento por explosión con vapor empleando un factor de severidad de 3,3 reduce el contenido de lignina evaluada como lignina klason de la cascarilla de arroz por un proceso de depolimeración verificado por la reducción de la transmitancia de los principales grupos funcionales asociados a la lignina y cambios estructurales de la superficie evaluados en el SEM. Esta modificiación de la superficie mejora el potencial de hidrólisis de la cascarilla de arroz pretratada para la formación de azúcares reductures que pueden ser aprovechadas en la producción de etanol G-2.

\section{AGRADECIMIENTO}

Se agradece al Programa Nacional de Innovación Agraria - PNIA, Fondo Concursable Investigación Estratégica Priorizada del concurso N ${ }^{\circ}$ 2-IE-PNIA-2016, patrocinadores del Proyecto Producción de etanol de 2 da generación G-2 a partir de residuos agroindustriales de caña, arroz y maíz, por su apoyo en el desarrollo de la presente investigación.

\section{REFERENCIAS BIBLIOGRÁFICAS}

1. Sistema Integrado de Estadística Agraria, SIEA. Anuario Estadístico de la producción Agrícola y Ganadera 2015. Lima, Perú: Ministerio de Agricultura y Riego; 2016.

2. Zou Y, Yang T. Chapter 9 - Rice Husk, Rice Husk Ash and Their Applications. Rice Bran and Rice Bran Oil, Chemistry, Processing and Utilization. 1st edition. Urbana, Illinois (USA): AOCS Press/Academic Press; 2019, pag: 207-246.

3. Kumar P, Barrett DM, Delwiche MJ, Stroeve P. Methods for pretreatment of lignocellulosic biomass for efficient hydrolysis and biofuel production. Ind Eng Chem Res. 2009; 48: 3713-3729.

4. Nitsos C, Matsakas L, Triantafyllidis K, Rova U, Christakopoulos P. Investigation of different pretreatment methods of Mediterranean-type ecosystem agricultural residues: characterisation of pretreatment products, high-solids enzymatic hydrolysis and bioethanol production. Biofuels. 2018; 9(5): 545-558.

5. Matsakas L, Raghavendran V, Yakimenko O, Persson G, Olsson E, Rova U, et al. Ligninfirst biomass fractionation using a hybrid organosolv - steam explosion pretreatment technology improves the saccharification and fermentability of spruce biomass. Bioresour Technol. 2019; 273: 521-528.

6. Fu C, Mielenz J, Xiao X, Ge Y, Hamilton CY, Rodriguez JM, et al. Genetic manipulation of lignin reduces recalcitrance and improves ethanol production from switchgrass. Proc Natl Acad Sci USA. 2011; 108: 3803-3808.

7. Wang Y, Gong X, Hu X, Zhou N. Lignin monomer in steam explosion assist chemical treated cotton stalk affects sugar release. Bioresour Technol. 2019; 276: 343-348.

8. Pereira L. The chemistry involved in the steam treatment of lignocellulosic materials. Quim Nova. 2003; 6: 863-871. 
9. Bonfiglio F, Cagno M, Rey F, Torres M, Böthig S, Mendez P, et al. Pretreatment of switchgrass by steam explosión in a semi-continuos pre-pilot reactor. Biomass Bionerg. 2019; $12: 41-47$.

10. Moniruzzaman M. Effect of Steam Explosion on the Phisicochemical Properties and Enzymatic Saccharification of Rice Straw. Appl Biochem Biotechnol. 1996; 59(3): 283297.

11. Chua MGS, Wayman M. Characterization of Autohydrolysis Aspen (P. tremuloides) Lignins. Part 1. Composition and Molecular Weight Distribution of Extracted Autohydrolysis Lignin. Can J Chem. 1979; 57: 1141-1149.

12. Tomczak F, Satyanarayana KG, Sydenstricker THD. Studies on lignocellulosic fibers of Brazil: part III e morphology and properties of Brazilian curaua fibers. Compos A Appl Sci Manuf. 2007; 38: 2227 - 2236.

13. Brígida AIS, Calado VMA, Gonçalves LRB, Coelho MAZ. Effect of chemical treatments on properties of green coconut fiber. Carbohydr Polym. 2010; 79: 832 - 838.

14. Romanzini D, Ornaghi HL, Amico SC, Zattera AJ. Preparation and characterization of ramie-glass fiber reinforced polymer matrix hybrid composites. Mater Res. 2012; 15 : $415-420$.

15. Boonterm M, Sunyadeth S, Dedpakdee S, Athichalinthorn P, Patcharaphun S, Mungkung $\mathrm{R}$, et al. Characterization and comparison of cellulose fiber extraction from rice straw by chemical treatment and thermal steam explosion. J Clean Prod. 2016; 134 (Part B): $592-599$.

16. Fang M, Yang L, Chen G, Shi Z, Luo Z, Cen K. Experimental study on rice husk combustion in a circulating fluidized bed. Fuel Process. Technol. 2004; 85: 1273-1282

17. Rodríguez M. Determinación de la Composición Química y Propiedades Físicas y Químicas del Pulido de Arroz (Oryza sativa L.). [Tesis Ciencia de los Alimentos]. Valdivia: Universidad Austral de Chile; 2007.

18. Valverde A, Sarria B, Monteagudo J. Análisis comparativo de las características fisicoquímicas de la Cascarilla de arroz. Scientia et Technica. 37(1): 255-260. doi: $10.22517 / 23447214.4055$

19. De Oliveira J, Pinheiro G, Oliveira K, Mello S, Silveira G, Guerra A, et al. da Rosa E. Cellulose fibers extracted from rice and oat husk and their application in hidrogel. Food Chem. 2017; 221: 153 - 160.

20. Quispe I, Navia R, Kahhat R. Energy potential from rice husk through direct combustion and fast pyrolysis: a review. Waste Manag. 2017; 59: 200-210.

21. Dekker R, Wallis A. Enzymic Saccharification of Sugarcane Bagasse Pretreated by Autohydrolysis - Steam Explosion. Biotechnol Bioeng. 1983; 25(12): 3027-3048.

22. Genieva SD, Turmanova SC, Dimitrova AS, Vlaev LT. Characterization of rice husks and the productions of its thermal degradation in air or nitrogen atmosphere. J. Therm. Anal. Calorim. 2008; 93: 387-396. 